\title{
RESPONSE OF THE RF POWER SYSTEM TO OFF-NORMAL CONDITIONS ON APT ${ }^{*}$
}

\author{
M. McCarthy", T. Overett, J. Tooker, General Atomics, San Diego, CA \\ M. Lynch, D. Rees, A. Regan, T. Wangler, LANL, Los Alamos, NM
}

\begin{abstract}
The Accelerator Production of Tritium (APT) plant utilizes a $1700 \mathrm{MeV}$ linear accelerator. The accelerating potential for the $100 \mathrm{~mA}$ proton beam is provided by up to (depending on production requirements) 244 one MW klystrons. Control of amplitude and phase at each coupling port provides optimum beam parameters. However, when a fault occurs or operation is required at reduced beam currents ('off-normal conditions'), the Radio Frequency (RF) system must compensate to maintain the cavity field. This paper will describe the projected off-normal conditions and the response of the RF system to accommodate these conditions and minimize downtime.
\end{abstract}

\section{INTRODUCTION}

There are three distinct sections of the linac with respect to RF power coupling [1]. These are: 1) the Radio Frequency Quadrupole (RFQ), which immediately follows the injector and serves primarily to bunch the beam $(6.7 \mathrm{MeV}) .2$ ) The Coupled Cavity Drift Tube Linac (CCDTL) and Coupled Cavity Linac (CCL) modules, which accelerate the beam in normal conducting (NC) cavities up to $211 \mathrm{MeV}$. There are eleven of these type modules. Except for the first module, which has only one klystron driving it, the other ten have between three and seven klystrons driving them. The term 'supermodule' has been given to a module with synchronous multiple klystron drives. 3) The cryomodule section, which uses superconducting (SC) cavities, accelerates the beam up to $1700 \mathrm{MeV}$. Under normal operating conditions with 100 $\mathrm{mA}$ of beam, the RF power is well matched to the linac cavities and little power is reflected back through the waveguides. However, before achieving normal operating conditions, the cavities must be brought up to full RF accelerating field strength with no beam present. All klystrons are ramped up to their design operating power of $80-90 \%$ of maximum. To ameliorate AC power draw on the utility grid, the klystrons are brought up gradually in groups. Because of the nature of accelerator operations, the RF Power Distribution System must have loads sized to handle reflected power under no-beam conditions continuously. With no beam the RFQ reflects $4 \%$ (VSWR $=1.5$ ) of the incident RF power and similarly, the CCDTL/CCL (VSWR $=4)$ reflects $36 \%$. The superconducting modules have low loss, very high $\mathrm{Q}$ cavities that, without a beam to absorb the incident RF, reflect nearly all power back through the waveguides.

The length of time during linac commissioning or restart conditions that the cavities are sans beam can not be dictated by the RF System. The RF System must be able to absorb this reflected power continuously. Under nobeam conditions most of the reflected RF power travels back to the circulator load in the RFQ and NC sections. This occurs because the reciprocal nature of the waveguide power splitters. It is also a result of the relative reflected phase at the splitter arms being equal to the incident phase. In all of the NC linac sections, the cavities are driven in-phase; thus the reflected power is also in phase. However, in the SC section, the reflected power ( $1 \mathrm{MW})$ is absorbed in both the circulator load and in local splitter loads. The power in each is a function of the phase difference between the cavities in the SC module [2]. The sum of the power dissipated in the loads and dissipated in the linac cavities must equal the incident power from the klystrons.

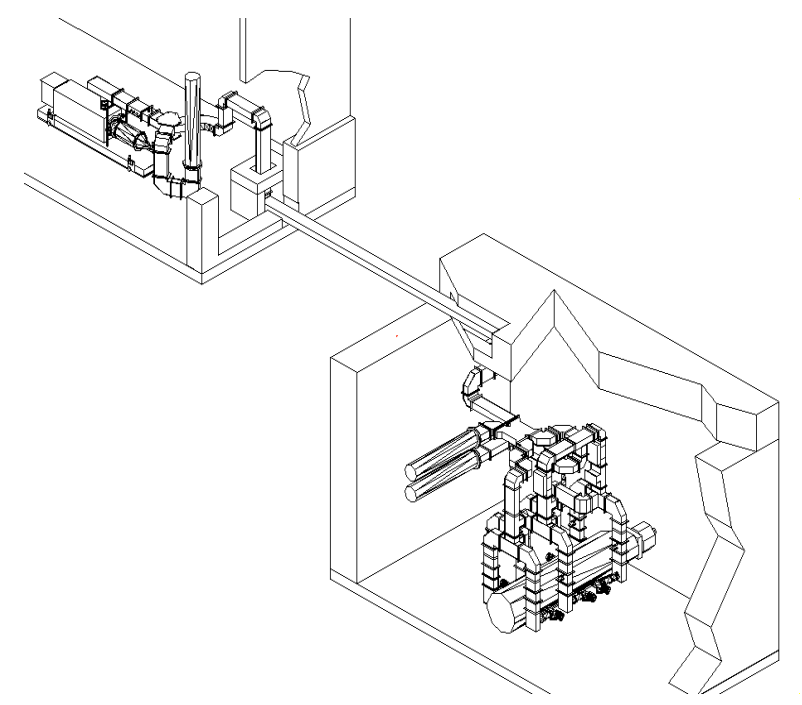

Figure 1: Typical RF power distribution from the klystron gallery to the linac tunnel.

In Figure 1, a pair of cylindrical high power RF watercooled loads can be seen in the tunnel and one in the klystron gallery. These loads share the total reflected power.

\footnotetext{
* Work supported by DOE contract DE-AC04-96AL89607.

** mccartm@gat.com
} 


\section{OFF-NORMAL DETECTION}

Before considering the response to various off normal conditions, the conditions themselves and their method of detection must be defined. Other than commissioning and re-start operations, all other off-normal conditions are the result of a system fault. Typical faults include RF arcs at the waveguide windows or cavities, window failures, RF System failures, high voltage power supply (HVPS) faults and beam loss resulting from injector, vacuum or other malfunctions.

\subsection{RF Diagnostics}

To detect an unexpected off-normal condition, the RF System has several diagnostics which run under the control of a low-level RF (LLRF) sub-system [3]. These diagnostics follow:

1)Fiber-optic (FO) lines are placed near the RF windows at the accelerating structure and in vacuum waveguide to view the flash from an arc. The FO lines carry light pulses back to optical detectors in racks on the mezzanine of the klystron gallery.

2)RF directional couplers are integrated in the waveguide transmission system near the cavity couplers and near the RF splitter loads. These couplers detect forward and reflected RF power levels. The signals are carried back to the mezzanine by standard coax cables for processing by the RF diagnostic system.

3)Air and surface temperature Resistance Temperature Devices (RTD's) or thermocouples. These devices will measure the outer surface of the window frame and the temperature of the cooing air that blows across the RF vacuum windows. These are excellent predictors of impending window failure. Cables are routed back to the klystron gallery to the RF diagnostic system.

\subsection{Low Level RF}

The LLRF system controls many functions, primarily the level of RF fields in the accelerating cavities. The LLRF controls the amplitude and phase of the klystrons by monitoring pick-ups from the linac and directional coupler feedback and reference signals running the length of the linac. It communicates with the Integrated Control System (ICS) to provide the status of the RF Systems to plant operations. The LLRF can issue a fast command to the injector in the event of a fault to terminate beam operations. It also interfaces with the RF diagnostics to evaluate a fault. The LLRF operates one RF power control function in response to a fault and that is the interruption of the RF drive to the klystron. The LLRF evaluates the fault and interrupts the drive for an appropriate amount of time. During the time the drive is off, all of the klystron power is dumped into the klystron's collector. The klystrons have been specified to withstand this power continuously. This feature is essential to the external AC utility grid [4] which would be seriously effected by 244 MW (worst case) suddenly turning off and on.

\section{SYSTEM SCENARIOS \& RESPONSES}

The following are off-normal operating modes and fault conditions and the corresponding RF Power and LLRF responses:

Start up scenario: All klystrons are brought up to full $\mathrm{CW}$ power $(<1 \mathrm{hr}$.) Reflected power is absorbed in RF loads. The beam duty factor is set very low $(<5 \mathrm{~Hz}, 100$ $200 \mu \mathrm{sec})$ and the steering, focusing, RF phase and amplitudes are optimized. The beam current is then increased to $100 \mathrm{~mA}$ at low duty and the phasing is rechecked. Once the accelerator is commissioned the duty factor is brought to $\mathrm{CW}$ at $100 \mathrm{~mA}$. At this point most of the RF power is absorbed in the linac cavities and beam and little is absorbed in the RF loads. [5]

Klystron power supply trip (crowbar). Action: The corresponding klystron shuts down. Beam is turned off to prevent beam spill, which would result from improper accelerating potentials in the cavities. If the klystron is part of a group that drives a supermodule, then all of the klystrons driving the supermodule are shut down to prevent RF induced power from coupling from the cavity to the waveguide. The power supply is reset and the klystron(s) brought back on line. The beam is restarted.

Waveguide or cavity arc. Action: The window arc detector sees the arc or the directional coupler of the waveguide associated with the arc detects high reflected power. The LLRF system shuts down the associated klystron(s) $(<10 \mu \mathrm{s})$. The LLRF also sends a signal to the injector to shut down the beam. The LLRF system can be programmed as to the time to restart the klystron drive after an arc and the number of arcs to permit before shutting down the klystron. The klystron is brought back up $(\sim 30 \mu \mathrm{sec})$ and the beam is restored. If the arc reoccurs and the RF is off for more than $500 \mathrm{~ms}$, the RFQ and NC structures will lose resonance. The LLRF system will go into a frequency agile mode and restore the structures to resonance. If the klystron is part of a group that drives a supermodule, then the RF drive to all the klystrons driving the supermodule are shut down to prevent RF induced power from coupling from the cavity to the waveguide and possibly maintaining an arc. The LLRF system restarts the klystron(s) after a predetermined time $(\sim 150 \mathrm{~ms})$.

RF System Failure: Action: Any RF System failure will interrupt the RF drive to the klystron. The LLRF system shuts down the beam. If the klystron is part of a group that drives a supermodule, then all of the klystrons driving the supermodule are shut down to prevent RF induced power from coupling from the cavity to the waveguide. A waveguide switch is thrown $(\sim 5 \mathrm{sec})$ on the failed system which causes the waveguide to present a 'short' to the cavity aperture. Therefore effectively no cavity power is lost. This switch will be located before the waveguide splits to the couplers and will function for 
all four couplers simultaneously. If an RF vacuum valve is employed, we will gain the option to operate with one of the two coupler-pairs with an increase in availability. The beam is restarted and the NC cavity structures retuned. If the klystron was part of a supermodule, the remaining klystrons on that module are brought up at full power to compensate for the lost klystron. If the failed klystron drives a superconducting (SC) module, that cavity is de-tuned ( $1 \mathrm{~min}$.) and the linac is returned to service. The succeeding SC modules are phase adjusted to compensate for the lost module. The medium beta and high beta SC modules are divided into four sections. The linac can lose a module in each of these sections and still meet operational requirements. The failed RF station can then be repaired while the linac continues to operate.

Window Failure: Thermal or arc sensors indicate impending window failure. Actions: For NC linac: shut down klystron and beam. Close waveguide switch. Restart beam. For SC linac: shut down klystron, detune cavity and restart beam.

Vacuum System failures, Injector failures, diagnostic failures, etc.: Failures in these categories may mean beam downtime in excess of 10 minutes for repair or while redundant systems are implemented. If the failure is judged to be in excess of 2-3 hours, RF Systems may be shut down to conserve power. There are many other possible failure modes. These will be identified and categorized as to the appropriate response. For the RF Power Distribution System, all failure modes need only be categorized into whether they cause a beam interruption and for how long. Table 1 summarizes the off-normal conditions, response, and expected downtime [6].

\section{CONCLUSION}

The RF system responds to off-normal conditions both passively (waveguide loads) and actively (LLRF control of klystron drive). It does so in a manner, which minimizes damage and downtime. The exact response and timing to a particular fault will be refined empirically as experience with this particular accelerator is acquired.

Table 1 The APT RF Power Distribution System under Off-Normal conditions.

\begin{tabular}{|c|c|c|c|c|c|}
\hline Off Normal Condition & RF System Response & Time & LLRF Response & Other Actions & $\begin{array}{l}\text { Down } \\
\text { time }\end{array}$ \\
\hline Start-up scenario & $\begin{array}{l}\text { Full power to cavities (no } \\
\text { beam) }\end{array}$ & $\begin{array}{l}\text { Hours- } \\
\text { days }\end{array}$ & \multicolumn{2}{|c|}{ Monitor phase of beam pulses. } & $\mathbf{N A}$ \\
\hline $\begin{array}{l}\text { Klystron power supply } \\
\text { trip (Crowbar). }\end{array}$ & \multicolumn{2}{|l|}{$\begin{array}{l}\text { Shut down klystron, reset PS, } \\
\text { Turn on klystron }\end{array}$} & \multicolumn{2}{|c|}{$\begin{array}{l}\text { Shut down beam, bring up beam after cavities } \\
\text { filled. }\end{array}$} & 5 min. \\
\hline $\begin{array}{l}\text { Klystron Waveguide Arc } \\
\text { or Cavity Arc }\end{array}$ & $\begin{array}{l}\text { Shut down klystron, Turn } \\
\text { on klystron }\end{array}$ & $150 \mathrm{~ms}$ & \multicolumn{2}{|c|}{ Detect arc. Kill beam. Interrupt klystron drive. } & $150 \mathrm{~ms}$ \\
\hline Klystron Failure & $\begin{array}{l}\text { Shut down klystrons, close } \\
\text { waveguide switch. }\end{array}$ & $10 \mu$ sec & $\begin{array}{l}\text { Detect failure. Shut down } \\
\text { beam, }\end{array}$ & $\begin{array}{l}\text { Other klystrons in } \\
\text { supermodule shut } \\
\text { down }\end{array}$ & $30 \mathrm{~min}$. \\
\hline $\begin{array}{l}\text { Impending Window } \\
\text { Failure }\end{array}$ & $\begin{array}{l}\text { Shut down associated } \\
\text { klystron, close WG switch }\end{array}$ & 5 sec. & $\begin{array}{l}\text { Detect thermal aberration } \\
\text { or arc. }\end{array}$ & $\begin{array}{l}\text { Other klystrons in } \\
\text { supermodule shut } \\
\text { down }\end{array}$ & 5 sec. \\
\hline Leaking Window & $\begin{array}{l}\text { Shut down klystron, close } \\
\text { WG switch }\end{array}$ & 5 sec. & $\begin{array}{l}\text { Shut down beam, close } \\
\text { gate valve, }\end{array}$ & $\begin{array}{l}\text { Actuate air and } \\
\text { vacuum valves. }\end{array}$ & $\begin{array}{l}\text { 5min.-24 } \\
\text { hrs. }\end{array}$ \\
\hline
\end{tabular}

\section{REFERENCES}

[1] Overview of the APT RF Power Distribution System, M. McCarthy, et al., LINAC'98.

[2] Based on cavity phase relationships provided by T. Wangler, LANL, April 8, 1998.

[3] LEDA LLRF Control System Performance: Model and Operational Experience, A. Regan, et al., PAC'99 Conference.
[4] Linear Accelerator Impact Study; K. Clark, et al., Sept., 1996, South Carolina Electric \& Gas.

[5] Development of a Commissioning Plan for the APT Linac, M. Schulze, et al., PAC'99 Conference.

[6] M. Lynch, Analysis of Options for Spare RF Systems for CCDTL-1, LANL memo\# LANSCE-5-98-010 Rev 0, Feb. 25, 1998. 